\title{
The Comparison between Random Forest and Support Vector Machine Algorithm for Predicting $\beta$-Hairpin Motifs in Proteins*
}

\author{
Shaochun Jia, Xiuzhen Hu, Lixia Sun \\ Department of Physics, College of Sciences Inner Mongolia University of Technology, Huhhot, China \\ Email: hh_xx_zz@yahoo.com.cn
}

Received 2013

\begin{abstract}
Based on the research of predicting $\beta$-hairpin motifs in proteins, we apply Random Forest and Support Vector Machine algorithm to predict $\beta$-hairpin motifs in ArchDB40 dataset. The motifs with the loop length of 2 to 8 amino acid residues are extracted as research object and the fixed-length pattern of 12 amino acids are selected. When using the same characteristic parameters and the same test method, Random Forest algorithm is more effective than Support Vector Machine. In addition, because of Random Forest algorithm doesn't produce overfitting phenomenon while the dimension of characteristic parameters is higher, we use Random Forest based on higher dimension characteristic parameters to predict $\beta$-hairpin motifs. The better prediction results are obtained; the overall accuracy and Matthew's correlation coefficient of 5-fold cross-validation achieve $83.3 \%$ and 0.59 , respectively.
\end{abstract}

Keywords: Random Forest Algorithm; Support Vector Machine Algorithm; $\beta$-Hairpin Motif; Increment of Diversity; Scoring Function; Predicted Secondary Structure Information

\section{Introduction}

$\beta$-hairpin is a super secondary structure motif. In the $\beta-\beta$ motif, if two anti-parallel $\beta$-strands are connected by loop and there are one or more hydrogen bonds between two adjacent strands, then the structure is called as $\beta$-hairpin [1-3], otherwise it is considered as non- $\beta$-hairpin. Correct prediction of $\beta$-hairpin motifs is helpful to folding recognition, and it is vital for simplifying folding numbers of unknown structure [4-6]. Therefore prediction of $\beta$-hairpin motifs has very important meaning.

In the past few years, some methods have been developed for predicting $\beta$-hairpin motifs in different datasets and better prediction results were obtained. In 2002, Cruz et al. [2] employed an artificial neural network (ANN) for predicting $\beta$-hairpins in 534 protein chains; an accuracy of $47.7 \%$ was obtained. In 2004, Kuhn et al. [1] also used ANN for predicting hairpins in 2209 protein chains by identifying local hairpins and non-local diverging turns; an accuracy of $75.9 \%$ was achieved. In 2005, Kumar et al. [3] used a Support Vector Machine (SVM) and ANN technique to predict $\beta$-hairpins in 2880 no redun-

*National Natural Science Foundation of China (30960090).

Natural Science Foundation of the Inner Mongolia of China (project No.2009MS0111).

Project for University of Inner Mongolia of China (project, NJZY08059). dant protein chains and obtained an accuracy of $79.2 \%$. In 2008, our group's Hu [7] et al. predicted $\beta$-hairpins in ArchDB40 dataset by using SVM, the overall accuracy and Matthew's correlation coefficients are $79.9 \%$ and 0.59, respectively. In 2010, our group's Hu et al. [8] attempted to use a quadratic discriminate (QD) method for predicting $\beta$-hairpins in ArchDB40 dataset, the overall accuracy and Matthew's correlation coefficients are 81.6\% and 0.55 , respectively.

In this article, we attempt to use a combination classifier algorithm, Random Forest and Support Vector Machine to predict $\beta$-hairpin motifs in ArchDB40 dataset. By using of the composite vector with increment of diversity, scoring function and predicted secondary structure information as characteristic parameters. When using Random Forest as prediction algorithm, the overall accuracy and Matthew's correlation coefficient of 5-fold cross-validation achieve $82.0 \%$ and 0.55 , respectively. However, when Support Vector Machine is used as prediction algorithm, they are only $79.4 \%$ and 0.49 , respectively. Similarly, the results of Random Forest algorithm are also better than Support Vector Machine for the independent test. Furthermore, we also use Random Forest based on higher dimension characteristic parameters to predict $\beta$-hairpin motifs. The prediction results are further improved. 


\section{Materials and Methods}

1) Materials

Our algorithm is trained and tested on ArchDB40 dataset. That is generated from ArchDB, in which the classification of protein loops from no redundant proteins of known structures

(http://www.sbi.imim.es/cgi-bin/archdb/loops.pl).

ArchDB was based on DSSP [9] database and provided by Oliva et al. [10,11]. ArchDB40 subset contains 3,088 no redundant proteins with resolution $<3.0 \AA$, in which no two protein chains have a percentage identity $>40 \%$ (ASTRAL SCOP 1.65). The ArchDB40 subset contains $9180 \beta$ - $\beta$ motifs are divided into $6216 \beta$-hairpin motifs and 2964 non- $\beta$-hairpin motifs. Here a total of $6028 \beta$ hairpin motifs and 2643 non- $\beta$-hairpin motifs with the loop length of 2 to 8 amino acid residues are selected as research object.

2) Methods

a) Random Forest (RF) Algorithm.

Random Forest that had been originally proposed by Leo Breiman [12] in 2001 is an ensemble classifier, it contains many decision trees. For each tree in the forest, a training set is firstly generated by randomly choosing $N$ times with replacement from all $N$ samples of the original dataset (bootstrap), and the rest are used as a testing set. When each node of single decision tree is splitting, the number of features used for splitting each node of decision tree $(m)$ is firstly specified. Then $m$ out of $M$ features are randomly selected and the best split attribute on these $m$ features is used to split the node, such that the impurity at each node of single decision tree is minimized and each tree in the forest fully grows without pruning. A Random Forest with $k$ decision trees is formed by repeating $k$ times as above procedure, and then the Random Forest is used to predict test data. The final classification results are decided by all the votes [13,14].

Random Forest has two most significant parameters, one is the number of features used for splitting each node of decision tree ( $m, m \ll M$ where $M$ is the total number of features), another parameter is the number of trees ( $k$ ). In this work, and $m$ is equal to $\sqrt{M}, k$ is equal to 500. Random Forest algorithm is implemented by using the package in R software [15] (http://www.r-project.org/). One obvious properties of the algorithm is that it doesn't produce overfitting phenomenon when the characteristic parameters of higher dimension are used.

b) Support Vector Machine (SVM) Algorithm.

The Support Vector Machine (SVM) is a promising binary classification method developed by Vapnik [16]. As a supervise machine learning technology, the algorithm had been used for many kinds of pattern recognition problems. In addition, because the algorithm of Support Vector Machine is a convex quadratic optimization problem, the local optimal solution is certainly the global optimal one. But other algorithms (such as ANN) don't have these features of SVM. In this paper, we use SVM to predict $\beta$-hairpin motifs. SVM has been widely used by transforming the input vector into a high-dimension Hilbert space and seeking a separating hyperplane in this space. The form of the decision function is:

$$
f(x)=\operatorname{sgn}\left[\sum_{i=1}^{N} \alpha_{i}^{*} y_{i} K\left(x_{i} \cdot x\right)+b^{*}\right]
$$

In this paper, we select the radial basis kernel function (RBF) $\left(K\left(x_{i} \cdot x\right)=\exp \left(-g\left\|x_{i}-x\right\|^{2}\right)\right)$. The optimal values of parameters $C$ and $g$ are default. SVM has been compiled into the software packages; we use libsvm-2.89 SVM software packages

(http://www.csie.ntu.edu.tw/ cjlin/libsvm).

3) The Selection of the Characteristic Parameters

a) The Selection of the Fixed-length Pattern

According to Hu's [7] ideas, $\beta$-hairpin motifs with the loop length of 2 to 8 amino acid residues in the dataset are extracted as research object and the fixed-length pattern of 12 amino acids are selected. Particular rules described as below:

i) The first amino acid (beginning) of loop locates the fifth position of the fixed-length pattern (5 - 12).

ii) End of loop locates the eighth position of the fixed-length pattern (8 - 12).

iii) The loop locates the center of fixed-length pattern. If the loop length is odd, the central coil is mapped and six residues (excluding the central coil) from the lefthand side and five residues from the right-hand side are taken. If not, the central two coils are mapped and five residues (excluding the central two coils) from both the sides were taken (Lr-12).

For above three rulers, if pattern length was $<12$, residues flanking the peptide in the amino acid sequence were appended at both the ends.

b) Amino acids component of position (A)

We statistically analyze the amino acid compositions at 12 positions of the fixed-length pattern of $\beta$-hairpin and non- $\beta$-hairpin motifs. The results show that conservation of position is stronger in the fixed-length sequence fragments. So amino acids component of position is extracted as sequence information. Because of the fixedlength pattern are generated using three rules, amino acids component of position is described as a vector of $21 \times$ 12 dimensions (21 denotes 20 amino acids and one terminal residue) for each ruler.

c) Hydropathy component of position (Q)

Because of protein structure is seriously influenced by hydropathy characteristics of amino acids. So hydropathy component of position is extracted as sequence information. Similarly, hydropathy component of position is described as a vector of $7 \times 12$ dimensions ( 7 denotes 6 hydropathy characteristics for amino acids and one ter- 
Table 1. Hydropathy characteristics for $\mathbf{2 0}$ amino acids.

\begin{tabular}{cccc}
\hline Classification & Amino acids & Classification & Amino acids \\
\hline Strongly hydrophilic or polar & R, D, E, N, Q, K, H & Proline & P \\
Strongly hydrophobic & L, I, V, A, M, F & Glycine & G \\
Weakly hydrophilic or Weakly hydrophobic & S, T, Y, W & Cysteine & C \\
\hline
\end{tabular}

minal residue) for each ruler, classification of hydropathy characteristics [17] for 20 amino acids are showed in Table 1.

d) Increment of Diversity (ID)

In the state space of $s$ dimension, the diversity measure for diversity sources $S:\left\{m_{1}, m_{2} \ldots m_{s}\right\}$ is defined as [18]:

$$
D(S)=M \log M-\sum_{i} m_{i} \log m_{i}
$$

In the same state space, increment of diversity between the source of diversity $X:\left\{n_{1}, n_{2}, \ldots n_{s}\right\}$ and $Y:\left\{m_{1}, m_{2} \ldots\right.$ $\left.m_{s}\right\}$ is defined as:

$$
\begin{aligned}
& I D(X, Y) \\
& =(M+N) \log (M+N)-\sum_{i}\left(m_{i}+n_{i}\right) \log \left(m_{i}+n_{i}\right) \\
& -M \log M-N \log N+\sum_{i} m_{i} \log m_{i}+\sum_{i} n_{i} \log n_{i}
\end{aligned}
$$

Here $N=\sum_{i} n_{i}, M=\sum_{i} m_{i}$. Amino acids component of position is selected as the basic parameter, and then constructs 2 diversity sources for $\beta$-hairpin and non- $\beta$ hairpin motifs. Because of the fixed-length pattern are generated using three rules, arbitrary sequence segments can obtain 6 ID values (ID (A)) which be calculated by Equation (3). Similarly, hydropathy component of position is also selected as the basic parameter. Arbitrary sequence segments obtain 6 ID values (ID (Q)) which be calculated by Equation (3).

e) Scoring function(S)

The position weight scoring function is a simple but effective forecast algorithm. Here we only calculate the scores of $\beta$-hairpin and non- $\beta$-hairpin motifs as characteristic parameters, the score of segment can be defined as [19]:

$$
\begin{gathered}
S=\sum_{i=1}^{12} C_{i}\left(w_{i j}-w_{i, \min }\right) \\
w_{i j}=\log \left(\sum_{i=1}^{12} C_{i j}\left(w_{i, \max }-w_{i, \min }\right)\right. \\
C_{i}=100 / \log 21\left(\sum_{j=1}^{21} P_{i j} \log p_{i j}+\log 21\right) \\
P_{i j}=\left(n_{i j}+\sqrt{N_{i}} / 21\right) /\left(N_{i}+\sqrt{N_{i}}\right)
\end{gathered}
$$

Where $j$ is amino acid $j$ or terminal residue, $N_{i}$ is the number of amino acids and terminal residue at the position $i, n_{i j}$ is the number of amino acid $j$ or terminal residue at the position $i, w_{i}$, min and $w_{i}$, max are the minimal and maximal values of position weight at the position $i$, respectively. $w_{i j}$ is the observed position weight at the position $i, C_{i}$ is the conservation index vector at position $i$. Amino acids component of position is selected as the basic parameter. Because of the fixed-length pattern are generated using three rules, arbitrary sequence segments can obtain $6 \mathrm{~S}$ values (S12 (A)) which be calculated by Equation (4).

f) Predicted secondary structure information (SS)

In the research of predicting $\beta$-hairpin motifs, literature $[2,3]$ had used predicted secondary structure information as the characteristic parameters; better prediction results were obtained. In order to improve the prediction effect, we also extract predicted secondary structure information. These are obtained by using the PHD [2] software, and are represented by a vector of 3 dimensions which are the frequency of predicted secondary structure ( $\alpha$-helix, $\beta$-sheet and coils).

\section{Results and Discussion}

1) Performance Measures

In order to evaluate the correct prediction rate and the reliability of a predictive method, we use the following standard measures. Accuracy of prediction ( Acc ); Matthew's correlation coefficient ( $M C C$ ); sensitivity of $\beta$-hairpin $\left(Q_{o(H)}\right)$; sensitivity of non- $\beta$-hairpin prediction $\left(Q_{o(N H)}\right)$; specificity of $\beta$-hairpin prediction $\left(Q_{p(H)}\right)$; specificity of non- $\beta$-hairpin $\left(Q_{p(N H)}\right)$ [7]; calculating formula as follow:

$$
\begin{gathered}
A c c=[(p+r) /(p+r+o+u)] \times 100 \% \\
M C C=[(p \times r)-(o \times u)] / \sqrt{(p+u)(p+o)(r+u)(r+o)}(9) \\
Q_{o(H)}=[p /(p+u)] \times 100 \% \\
Q_{o(N H)}=[r /(r+o)] \times 100 \% \\
Q_{p(H)}=[p /(p+o)] \times 100 \%
\end{gathered}
$$




$$
Q_{p(N H)}=[r /(r+u)] \times 100 \%
$$

Here $p$ and $r$ denote the number of correctly predicted $\beta$-hairpin and non- $\beta$-hairpin, respectively; $u$ denotes the number of the $\beta$-hairpin that are predicted as non- $\beta$-hairpin, $o$ denotes the number of the non- $\beta$-hairpin that are predicted as $\beta$-hairpin.

2) The Predictive Results Using of 5-fold Cross-validation

By using of the composite vector with increment of diversity (ID(A) + ID(Q)), scoring function (S12(A)) and predicted secondary structure information (SS) as characteristic parameters. When RF algorithm is applied to predict $\beta$-hairpins, for 5-fold cross-validation, Acc and MCC are $82.0 \%$ and 0.55 , respectively. However, when SVM is used as prediction algorithm, Acc and MCC are only $79.4 \%$ and 0.49 , respectively. Besides, the $Q_{o}\left({ }_{H}\right)$, $Q_{o}\left({ }_{N H}\right), Q_{p}(H)$, and $Q_{p}\left({ }_{N H}\right)$ of RF algorithm are all higher than SVM. The results show that RF algorithm is better than SVM. In addition, to compare our method with others, we also list previous prediction results in Table 2. It can be seen that our prediction overall accuracy of using RF algorithm is slightly higher than Hu's $[7,8]$ results. But the Matthew's correlation coefficient is lower than Hu's $[7,8]$ results.

3) The Predictive Results Using of the independent test

To further compare RF and SVM algorithm, we also use the independent test. The $1028 \beta$-hairpins and 643 non- $\beta$-hairpins are selected as training set from 6028 $\beta$-hairpins and 2643 non- $\beta$-hairpins, the remaining 5000 $\beta$-hairpins and 2000 non- $\beta$-hairpins are independent testing set. By using of the composite vector (ID (A) + ID(Q) $+\mathrm{S} 12(\mathrm{~A})+\mathrm{SS})$ as the characteristic parameters, when $\mathrm{RF}$ algorithm is applied to predict $\beta$-hairpin motifs. Acc and $M C C$ are $79.9 \%$ and 0.50 , respectively. However, when SVM algorithm is used as prediction algorithm, AcC and MCC are only $77.0 \%$ and 0.43 , respectively. Predictive results are showed in Table 3 . It can be seen that RF algorithm is still better than SVM. Furthermore, it should be noticed that using the small sample to test the large one in here.

4) The Predictive Results Using of higher dimension characteristic parameters

Considering the obvious properties of RF algorithm, we directly use the composite vector with amino acids component of position (A), hydropaths component of position (Q), and predicted secondary structure information (SS) as characteristic parameters (675 dimensions). Acc and MCC of 5-fold cross-validation achieve $83.3 \%$ and 0.59 , respectively. It needs to be pointed out that, amino acids component of position and hydropaths component of position are only based on the first two cutting rules (5 - 12, 8 - 12) in here. In contrast, we also use of the composite vector (ID(A) + ID(Q) + SS) as the characteristic parameters. Acc and MCC of 5-fold cross-validation are $79.5 \%$ and 0.49 , respectively. The predictive results are decreased. Then we use RF algorithm based on the composite vector $(\mathrm{A}+\mathrm{Q}+\mathrm{SS})$ to predict $\beta$-hairpin motifs. For the independent test (I (test)), Acc and MCC are $80.1 \%$ and 0.50 , respectively. Predictive results are showed in Table 4. The results indicate that 5-fold cross-validation and the independent test are similar when RF algorithm is used to predict $\beta$-hairpin motifs.

\section{Conclusion}

In this paper, the predictive results of using RF algorithm based on the composite vector $(A+Q+S S)$ are better than previous. From above results we can seen: 1) RF algorithm is better than SVM when the same characteristic parameters and the same test method are used; 2) Due

Table 2. Predictive results using of 5 -fold cross validation for $\beta$-hairpinsand non $\beta$-hairpins in ArchDB40 dataset.

\begin{tabular}{|c|c|c|c|c|c|c|}
\hline Method (parameters) & $Q_{o(H)}(\%)$ & $Q_{\mathrm{o}(\mathrm{NH})}(\%)$ & $Q_{p(H)}(\%)$ & $Q_{p(\mathrm{NH})}(\%)$ & Acc (\%) & MCC \\
\hline RF(ID(A), ID(Q), S12 (A), SS) & 92.0 & 59.2 & 83.7 & 76.4 & 82.0 & 0.55 \\
\hline SVM(ID(A), ID(Q), S12 (A), SS) & 89.1 & 57.3 & 82.6 & 69.7 & 79.4 & 0.49 \\
\hline SVM(S, ID) [7] & 80.3 & 79.3 & 86.1 & 71.5 & 79.9 & 0.59 \\
\hline QD(S12(a), ID(aa), ID(qq), ID $\left.\left(A_{f}\right)\right)[8]$ & 89.1 & 64.7 & 85.2 & 72.2 & 81.6 & 0.55 \\
\hline
\end{tabular}

Table 3. Predictive results using of the independent test for $\beta$-hairpins and non $\beta$-hairpins in ArchDB40 dataset.

\begin{tabular}{|c|c|c|c|c|c|c|}
\hline Method(parameters) & $\mathbf{Q}_{\mathrm{o}(\mathrm{H})}(\%)$ & $Q_{\mathrm{o}(\mathrm{NH})}(\%)$ & $Q_{p(H)}(\%)$ & $Q_{\mathrm{p}(\mathrm{NH})}(\%)$ & Acc (\%) & MCC \\
\hline RF(ID(A), ID(Q), S12(A), SS) & 91.2 & 54.1 & 81.9 & 72.9 & 79.9 & 0.50 \\
\hline SVM(ID(A), ID(Q), S12(A), SS) & 87.9 & 52.1 & 80.7 & 65.5 & 77.0 & 0.43 \\
\hline
\end{tabular}

Table 4. Predictive results using Random Forest algorithm for $\beta$-hairpins and non $\beta$-hairpins in ArchDB40datase.

\begin{tabular}{ccccccccc}
\hline & parameters & dimension & $\mathbf{Q}_{\mathbf{0}(\mathbf{H})} \mathbf{( \% )}$ & $\mathbf{Q}_{\mathbf{o}(\mathrm{NH})} \mathbf{( \% )}$ & $\mathbf{Q}_{\mathbf{p ( H )}} \mathbf{( \% )}$ & $\mathbf{Q}_{\mathbf{p}(\mathrm{NH})} \mathbf{( \% )}$ & Acc (\%) & $\mathbf{M C C}$ \\
\hline \multirow{2}{*}{ 5-fold } & ID(A) + ID(Q) + SS & 15 & 90.8 & 53.5 & 81.7 & 71.9 & 79.5 & 0.49 \\
& A + Q + SS & \multirow{2}{*}{675} & 95.4 & 55.9 & 83.1 & 84.1 & 83.3 & 0.59 \\
I (test) & A + Q + SS & & 91.2 & 54.7 & 82.1 & 73.1 & 80.1 & 0.50 \\
\hline
\end{tabular}


to RF algorithm doesn't produce overfitting when the dimension of the characteristic parameters is higher, better results are still obtained. But the phenomenon will appear in this case for the SVM; 3) Previous independent test is usually that using the large sample to test the small one. However, we still obtain better predictive results by using the small sample to test the large one when RF algorithm is used. This implies that RF algorithm is steady and effective.

\section{REFERENCES}

[1] M. Kuhn, J. Meiler and D. Baker, "Strand-Loop-Strand Motifs: Prediction of Hairpins and Diverging Turns in Proteins," Proteins: Structure, Function, and Bioinformatics, Vol. 54, 2004, pp. 282-288.

http://dx.doi.org/10.1002/prot.10589

[2] X. Cruz, E. G. Hutchinson, A. Shepherd and J. M. Thornton, "Toward Predicting Protein Topology: An Approach to Identifying $\beta$ Hairpins," Proceedings of the National Academy of Sciences of the USA, Vol. 99, 2002, pp. 11157-11162. http://dx.doi.org/10.1073/pnas.162376199

[3] M. Kumar, M. Bhasin, N. K. Natt and G. P. S. Raghava, "BhairPred: Prediction of $\beta$-Hairpins in a Protein from Multiple Alignment Information Using ANN and SVM Techniques,” Nucleic Acids Research, Vol. 33, 2005, pp. 154-159. http://dx.doi.org/10.1093/nar/gki588

[4] T. F. Jenny, D. L. Gerloff, M. A. Cohen and S. A. Benner, "Predicted Secondary and Super Secondary Structure for the Serine-Threonine-Specific Protein Phosphatase Family," Proteins: Structure, Function, and Bioinformatics, Vol. 21, 1995, pp. 1-10.

[5] A. Godzik, J. Skolnick and A. Kolinski, "Simulations of the Folding Pathway of Triose Phosphate Isomerase-Type Alpha/Beta Barrel Proteins," Proceedings of the National Academy of Sciences of the USA, Vol. 89, 1992, pp. 2629-2633. http://dx.doi.org/10.1073/pnas.89.7.2629

[6] R. T. Wintjens, M. J. Rooman and S. J. Wodak, “Automatic Classification and Analysis of Alpha Alpha-Turn Motifs in Proteins," Journal of Molecular Biology, Vol. 255, 1996, pp. 235-253.

[7] X. Z. Hu and Q. Z. Li, "Prediction of the $\beta$-Hairpins in Proteins Using Support Vector Machine,” Protein Journal, Vol. 27, 2008, pp. 115-122. http://dx.doi.org/10.1007/s10930-007-9114-z

[8] X. Z. Hu, Q. Z. Li and C. L. Wang, "Recognition of $\beta$ Hairpin Motifs in Proteins by Using the Composite Vec- tor,” Amino Acids, Vol. 38, 2010, pp. 915-921. http://dx.doi.org/10.1007/s00726-009-0299-7

[9] W. Kabsch and C. Sander, "Dictionary of Protein Secondary Structure: Pattern Recognition of Hydrogen-Bonded and Geometrical Features,” Biopolymers, Vol. 22, 1983, pp. 2577-2637. http://dx.doi.org/10.1002/bip.360221211

[10] B. Oliva, P. A. Bates, E. Querol, F. X. Aviles and M. J. E. Sternberg, "An Automated Classification of the Structure of Protein Loops," Journal of Molecular Biology, Vol. 266, 1997, pp. 814-830. http://dx.doi.org/10.1006/jmbi.1996.0819

[11] J. Espadaler, N. F. Fuentes, A. Hermoso, E. Querol, F. X. Aviles, M. J. E. Sternberg and B. Oliva, “ArchDB: Automated Protein Loop Classification as a Tool for Structural Genomics,” Nucleic Acids Research, Vol. 32, 2004, pp. 185-188. http://dx.doi.org/10.1093/nar/gkh002

[12] L. Breiman, "Random Forests,” Machine Learning, Vol. 45, 2001, pp. 5-32. http://dx.doi.org/10.1023/A:1010933404324

[13] F. S. Edelenyi, L. Goumidi and S. Bertrais, "Prediction of the Metabolic Syndrome Status Based on Dietary and Genetic Parameters, Using Random Forest,” Genes \& Nutrition, Vol. 3, 2008, pp. 173-176. http://dx.doi.org/10.1007/s12263-008-0097-y

[14] O. Okun and H. Priisalu, "Random Forest for Gene Expression Based Cancer Classification: Overlooked Issues," Pattern Recognition and Image Analysis, Vol. 4478, 2007, pp. 483-490.

http://dx.doi.org/10.1007/978-3-540-72849-8_61

[15] A. Liaw and M. Wiener, "Classification and Regression by Random Forest,” R News, Vol. 2, 2002, pp. 18-22.

[16] V. Vapnik, “Statistical Learing Theory,” Wiley-Interscience, 1998.

[17] J. Panek, I. Eidhammer and R. Aasland, “A New Method for Identification of Protein (sub) Families in a Set of Proteins Based on Hydropathy Distribution in Proteins," Proteins: Structure, Function, and Bioinformatics, Vol. 58, 2005, pp. 923-934. http://dx.doi.org/10.1002/prot.20356

[18] R. R. Laxton, “The Measure of Diversity,” Journal of Theoretical Biology, Vol. 70, 1978, pp. 51-67. http://dx.doi.org/10.1016/0022-5193(78)90302-8

[19] J. M. Claverie and S. Audic, "The Statical Significance of Nucleotide Position-Weight Matrix Matches,” CABIOS, Vol. 12, 1996, pp. 431-439. 